\title{
Down Syndrome: Genes, Model Systems, and Progress towards Pharmacotherapies and Clinical Trials for Cognitive Deficits
}

\author{
J. Busciglio ${ }^{a}$ G. Capone ${ }^{b} \quad$ J.P. O'Byran ${ }^{c}$ K.J. Gardiner ${ }^{d}$ \\ a University of California Irvine, Irvine, Calif., ${ }^{b}$ Kennedy Krieger Institute, Baltimore, Md., ' University of Illinois at \\ Chicago, Chicago, III., and ' University of Colorado School of Medicine, Aurora, Colo., USA
}

\section{Key Words}

Chromosome HSA21 · Cognition · Down syndrome · Gene function · Mouse models · Trisomy

\begin{abstract}
Down syndrome (DS) is caused by an extra copy of all or part of the long arm of human chromosome 21 (HSA21). While the complete phenotype is both complex, involving most organs and organ systems, and variable in severity among individuals, intellectual disability (ID) is seen in all people with DS and may have the most significant impact on quality of life. Because the worldwide incidence of DS remains at approximately 1 in 1,000 live births, DS is the most common genetic cause of ID. In recent years, there have been important advances in our understanding of the functions of genes encoded by HSA21 and in the number and utility of in vitro and in vivo systems for modeling DS. Of particular importance, several pharmacological treatments have been shown to rescue learning and memory deficits in one mouse model of DS, the Ts65Dn. Because adult mice were used in the majority of these experiments, there is considerable interest in extending the studies to human clinical trials, and a number of trials have been completed, are in progress or are being planned. A recent conference brought together researchers
\end{abstract}

with a diverse array of expertise and interests to discuss (1) the functions of HSA21 genes with relevance to ID in DS, (2) the utility of model systems including Caenorhabditis elegans, zebrafish and mouse, as well as human neural stem cells and induced pluripotent stems cells, for studies relevant to ID in DS, (3) outcome measures used in pharmacological treatment of mouse models of DS and (4) outcome measures suitable for clinical trials for cognition in adults and children with DS.

(c) 2013 S. Karger AG, Basel

A brief overview of the number of genes encoded by human chromosome 21 (HSA21), their conservation in mouse, and their representation in segmental trisomy mouse models of Down syndrome (DS) was provided by Katheleen Gardiner (University of Colorado School of Medicine). Orthologs of HSA21 genes are dispersed among 3 mouse (MMU) chromosomes: the telomeric region of MMU16, and internal segments of MMU17 and

Report on the Conference on Cognition in Down Syndrome: Molecular, Cellular and Behavioral Features and the Promise of Pharmacotherapies. Washington D.C., April 13-15, 2013

\section{KARGER}

E-Mail karger@karger.com

www.karger.com/cgr
(C) 2013 S. Karger AG, Base

$1424-8581 / 13 / 1414-0260 \$ 38.00 / 0$
Katheleen J. Gardiner

Linda Crnic Institute for Down Syndrome

University of Colorado School of Medicine, Mail Stop 8608

$12700 \mathrm{E}$ 19th Avenue, Aurora, CO 80045 (USA)

E-Mail katheleen.gardiner@ucdenver.edu 


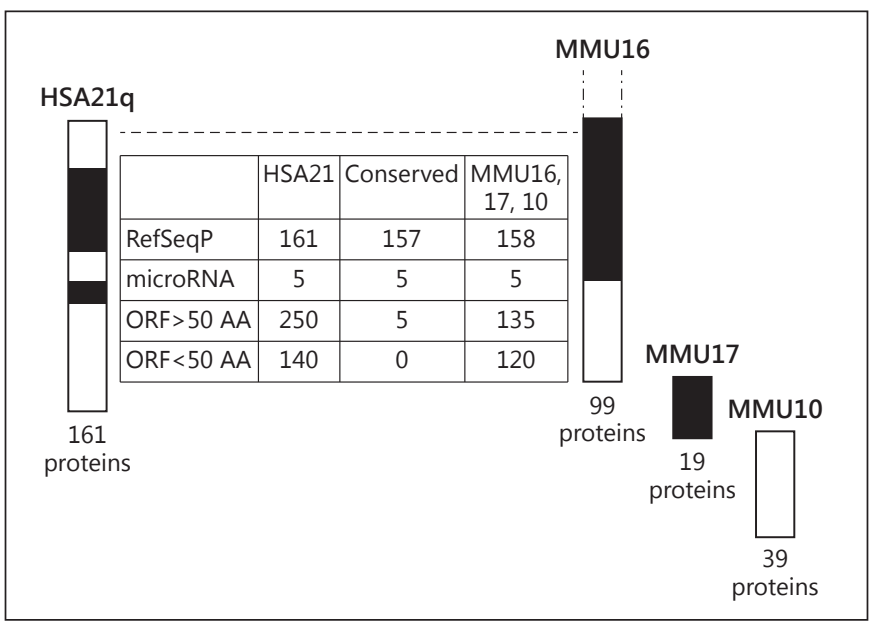

Fig. 1. Numbers of genes within HSA21 and orthologous mouse (MMU) chromosomal regions. RefSeqP = Protein-coding genes curated in the Reference Sequence Database (NCBI). Total numbers of protein-coding, RNA and novel genes are provided for human and mouse genomic regions. The number of conserved protein-coding genes is shown below each chromosome [Sturgeon and Gardiner, 2011]. AA $=$ Amino acid; ORF $=$ open reading frame.

MMU10. A recent comparative annotation and manual review of the gene content of HSA21 and orthologous mouse chromosomal regions showed that, if the $>40$ keratin-associated protein (KRTAP) genes are excluded, HSA21 encodes 161 unambiguous protein-coding genes and 5 microRNAs. Of these, 157 protein-coding genes and the microRNAs are conserved in mouse [Sturgeon and Gardiner, 2011], consistent with the major annotation systems (National Center for Biotechnology Information, http://www.ncbi.nlm.nih.gov/gene/; UniProtKB/Swiss-Prot, http://www.uniprot.org/; Ensembl Browser, http://www.ensembl.org/). In the DS/HSA21 literature, however, there appears to be either uncertainty or divergence of opinion regarding the total number of genes encoded by HSA21. The source of this confusion rests with species-specific genes. These are defined as experimentally verified, spliced human or mouse mRNAs whose exons are associated with consensus splice sites but lack nucleotide similarity to DNA or RNA sequences mapping in the genome of the other species. Some of these genes encode significant open reading frames, others are predicted to be functional RNA genes, and still others are not possible to classify. Species-specific genes have all the hallmarks of bona fide genes; they appear in the Ensembl Browser and are curated by the Vertebrate Genome Annotation (VEGA) system, which does not re-

Conference on Cognition in Down Syndrome quire evolutionary conservation in annotating gene structures [Wilming et al., 2008]. The total number of speciesspecific genes within HSA21 or mouse genomic regions varies with annotation system because each system has its own requirement for experimental support for a new gene. Regardless of criteria, however, both HSA21 and mouse orthologous regions have hundreds of speciesspecific genes. It is misleading simply to quote the total number of genes within an HSA21 region or a mouse segment. To be consistent between species and among annotation methods, the numbers of manually reviewed, conserved protein-coding genes is a good comparator. These are illustrated in figure 1.

Segmental trisomy mouse models of DS discussed at this conference, and their conserved gene content, are illustrated in figure 2. It is important to note that the popular Ts65Dn, in addition to being trisomic for orthologs of 88 HSA21 protein-coding genes, was recently determined to be trisomic for $\sim 50$ protein-coding genes (excluding pseudogenes) from a non-HSA21 orthologous centromere proximal segment of MMU17 [Duchon et al., 2011]. A list of conserved protein-coding genes on HSA21, in the Tc1 and in the Ts65Dn model is available in Ahmed et al. [2013, supplementary table 6]. Trisomic gene content is an important consideration in predicting how well, or if, observations in any DS mouse model, including drug responses, can be extended to human DS.

\section{The Genes of HSA21, Pathways and Processes They Influence}

\section{TRPM2}

Michael Jackson (Western University) discussed his work on the transient receptor potential $\mathrm{M} 2 \mathrm{Ca}^{2+}$ channel (TRPM2) that encodes channel forming domains and a Nudix-like domain. Binding of ADP-ribose (ADPR) regulates gating of the channel. TRPM2 mediates neuronal cell death in response to oxidative stress, suggesting a potential significant role in DS where oxidative stress has been a hallmark. Jackson showed that TRPM2 is expressed in CA1 pyramidal cells but not in GABAergic interneurons and that activation requires both ADPR binding and coincident entry of $\mathrm{Ca}^{2+}$ through voltage-gated or NMDA receptor channels. Using $T r p m 2^{-l-}$ mice, his group demonstrated that TRPM2 was required for NMDA receptor-dependent long-term depression (LTD). This effect was due to an increase in GSK3 $\beta$ phosphorylation at Ser9 resulting in decreased expression of PSD-95 and the AMPA receptor subunit GluR1 [Xie et 
Fig. 2. Down syndrome mouse models and HSA21 genes discussed in the text. A schematic of the long arm of HSA21, the Tc1 transchromosomal mouse and segmental trisomy mouse models are shown. The locations of single genes discussed in functional studies or as single gene transgenic mice are indicated. Numbers in parentheses indicate the number of conserved protein-coding genes within each segmental trisomy. Grey circles in the Tc1 chromosome indicate deleted regions. The striped segment in the Ts65Dn chromosome indicates the presence of a centromeric region of mouse chromosome 17 that is non-orthologous to HSA21. * = Genes discussed in Schupf et al. [2010]. See text for references.

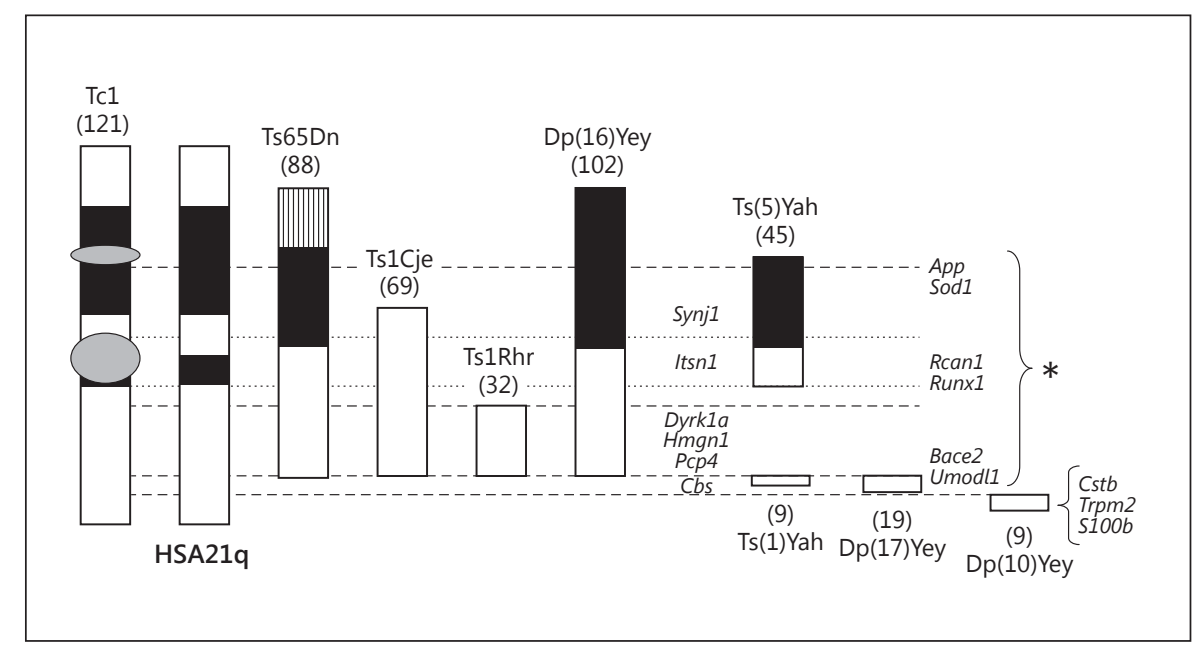

al., 2011]. Jackson speculated that elevation of TRPM2 in DS due to gene triplication could lead to increased GSK3 $\beta$ activation and increased LTD. Trpm2 maps to MMU10 and thus is not trisomic in the popular Ts65Dn mouse model (fig. 2). However, in a full trisomy HSA21, overexpression of TRPM2 could exacerbate abnormalities in LTD seen in the Ts65Dn.

\section{S100B}

Patricia Whitaker-Azmitia (Stony Brook University) presented work on S100B, a $\mathrm{Ca}^{2+}$-binding, EF-hand protein also mapping to the telomeric region of HSA21 and MMU10. S100B is expressed predominantly in astroglial cells with an age-dependent profile, and is overexpressed in DS and Alzheimer's disease (AD). Whitaker-Azmitia presented data showing that transgenic mice overexpressing human S100B from its endogenous promoter display increased repetitive behavior and impaired performance in the Morris water maze, harm avoidance and social interaction tests. Whitaker-Azmitia suggested that excess $\mathrm{S100B}$, as seen in DS, may accelerate the aging process. Compared to controls, S100B transgenic mice have a greater number of serotonin terminals at early ages but decreased numbers by 28 weeks [Shapiro et al., 2010]. A similar age-related decrease is observed in dendritic development. Older transgenic mice also show elevated microglial activation and increased levels of apoptotic markers, consistent with elevated neuroinflammation and cell death. Lastly, S100B transgenic mice have increased levels of Tau phosphorylation with an increase in plaques. Together, these observations suggest that $\mathrm{S} 100 \mathrm{~B}$ may contribute to phenotypic features seen in full trisomy HSA21.
If so, the increased expression of S100B may influence the phenotypic features and/or drug responses that are seen in the Ts65Dn.

\section{$A P P$ and $C S T B$}

Ralph Nixon (Nathan S. Kline Institute and New York University) described the involvement of the amyloid precursor protein (APP) in the phenotype of endosome dysfunction, one of the earliest disease markers in $\mathrm{AD}$ and in $\mathrm{AD}$ in $\mathrm{DS}$. In the Ts65Dn mouse, 3 copies of $A p p$ are necessary for the observed endosome enlargement as well as for the degeneration of the basal forebrain cholinergic neurons (BFCN). The phenotype is independent of $\mathrm{A} \beta$ but dependent on production of $\beta \mathrm{CTF}$, the $\mathrm{COOH}$ terminal fragment of APP produced by $\beta$-secretase (BACE1) cleavage. Overexpression of wild type (WT) $\mathrm{APP}$, but not a BACE1-resistant mutant $\left(\mathrm{APP}_{\mathrm{M} 596 \mathrm{~V}}\right)$, induces endosome enlargement. Genetic deletion of 1 copy of Bace1 in Ts65Dn rescues the endosome defects and restores BFCN numbers. In contrast, inhibition of $\gamma$-secretase increases $\beta$ CTF levels and worsens the endosomal phenotype. HSA21 genes in addition to APP are required for the endosome phenotype based on observations with the APP-YAC single gene transgenic mice.

Nixon also discussed the link between AD and autophagy which is important for normal turnover of organelles and long-lived proteins. In the $\mathrm{AD}$ brain, there is defective clearance of autophagy substrates and dystrophic neurites are filled with autophagy vesicles. Inhibition of lysosomal proteolysis induces AD-like defects and, conversely, preventing autophagy defects is protective. Although increased APP gene dosage contributes to defects 
in late endosome and lysosomal proteolytic function in DS fibroblasts, impaired autophagy induction is APP-independent suggesting involvement of additional HSA21 genes. Nixon discussed work from his group suggesting a contribution from the HSA21-encoded cysteine protease inhibitor cystatin B (CSTB). Genetic deletion of CSTB partially restored lysosomal proteolytic activity resulting in enhanced clearance of $A \beta$, ubiquitinated proteins, and other autophagy substrates [Yang et al., 2011]. In addition, loss of CSTB partially reversed the cognitive deficits and neuropathology in a mouse model of AD. Therefore, increased expression of CSTB in DS may contribute to AD in DS. Because CSTB maps to distal HSA21 and MMU10, this is further evidence for the need to consider genes not trisomic in the Ts65Dn.

Nataša Kopitar-Jerala (Jozef Stefan Institute) also discussed CSTB, particularly its role in inflammasome activation and cytokine secretion. Her previous work demonstrated a nuclear role for CSTB in interaction with nucleosomes through histones H2A.Z, H2B and H3 [Ceru et al., 2010]. Fibroblasts from $\mathrm{Cstb}^{-/-}$mice entered $S$ phase more rapidly than WT cells. Conversely, overexpression of CSTB delayed cell cycle progression. $\mathrm{Cstb}^{-/-}$mice are also characterized by increased microglia activation and increased cerebellar apoptosis which is thought to be mediated by cathepsin B. Given the link between CSTB and cathepsin B, coupled with the involvement of cathepsin B in inflammasome activation, Kopital-Jerala examined inflammasome activation in bone marrow-derived macrophages (BMM) from WT and Cstb ${ }^{-/-}$mice. CSTB is upregulated in activated macrophages. $\mathrm{Cstb}^{-/-} \mathrm{BMM}$ have increased caspase 1 and caspase 11 activation, increased pro-IL- $1 \mathrm{~b}$ processing and release of IL- $1 \mathrm{~b}$, and decreased IL-10 levels. Analysis of BMM revealed decreased ERK and JNK MAPK, decreased STAT activation, and elevation in nitric oxide levels likely due to elevation in iNOS induction in $\mathrm{Cstb}^{-/-}$macrophages versus WT cells.

Karl Pfenninger (University of Colorado School of Medicine) discussed additional properties of APP. Pfenninger found that APP levels were elevated 1.7-fold in axonal growth cones of Ts65Dn mice. To determine whether APP could function as a bona fide guidance molecule, he utilized synthetic, monospecific peptide substrates to selectively engage APP, $\beta 1$-integrin, or L1-CAM on cultured neurons overexpressing APP or $\beta 1$-integrin. His data demonstrated that APP functions as an adhesion molecule to guide axon growth [Sosa et al., 2013]. APP overexpressing neurons from Ts65Dn and DS fetal cortex preferred the APP-binding matrix, showed increased size, numbers of filopodia and adhesion. These findings

Conference on Cognition in Down Syndrome suggest that APP overexpression in DS may alter brain circuitry by perturbing growth cone guidance, neuron wiring and synaptic plasticity.

Nicole Schupf (Columbia University Medical Center, New York) described efforts to identify additional HSA21 genes contributing to the observed interindividual differences in A $\beta$ peptide levels in adults with DS [Schupf et al., 2010]. Genomic DNA from 261 nondemented adults with DS was examined for variants in APP and the HSA21 genes SOD1, DSCR1, RUNX1, BACE2, UMODL1, and $S 100 B$. Significant associations were observed with variants in APP, SOD1, DSCR1, RUNX1, and BACE2, but not $U M O D L 1$ or $S 100 B$. She proposed that these variants may serve as important biomarkers for prediction of $\mathrm{AD}$ risk in adults with DS.

\section{ITSN1}

John O'Bryan (University of Illinois at Chicago) discussed his work on the intersectin 1 (ITSN1) scaffold protein. ITSN1 is expressed in 2 major protein isoforms, ITSN1-short (S) and ITSN1-long (L), which differ only in the presence of the $\mathrm{Cdc} 42$ guanine nucleotide exchange factor domain on the COOH-terminus of ITSN1-L. He presented data demonstrating elevated expression of both isoforms in samples from DS patients. However, in patients over the age of 40 with DS and AD-like pathology, expression of both isoforms was decreased, suggesting a potential role for ITSN1 overexpression in neuron loss. Brain-specific overexpression of ITSN1-S in a novel transgenic model resulted in decreased locomotor behavior [Hunter et al., 2011]. O’Bryan also discussed the involvement of ITSN1 in oncogenesis. ITSN1 is expressed in neuroblastoma tumor cell lines and primary tumor samples. Loss of ITSN1 by infection of neuroblastoma cells with ITSN1-specific shRNA retrovirus decreased both anchorage-independent growth in soft agar in vitro and tumor growth in nude mice in vivo [Russo and O'Bryan, 2012]. Although the prevalence of neuroblastomas, as well as most solid tumors, is lower in DS patients compared to control populations, the incidence of hematopoietic malignancies in DS patients is 10-20-fold higher suggesting a potential link between ITSN1 overexpression in DS and cancer.

\section{DYRK1A}

Mara Dierssen (Center for Genomic Regulation, Barcelona) discussed her work on dual specificity Yak1-related kinase 1A (DYRK1A), a protein kinase encoded by HSA21 and overexpressed in DS. Much attention has centered on the importance of DYRK1A in the neuro- 
logical and cognitive deficits associated with DS. Using a DYRK1A transgenic mouse model (TgDYRK1A) which expresses approximately 1.5 -fold more DYRK1A compared to control mice, they observed decreased numbers of dendritic spines and reduced complexity of dendritic trees, similar to what is observed in the Ts65Dn model for DS. For both Ts65Dn and TgDYRK1A models, environmental enrichment leads to important but temporary improvements in cognitive function. Dierssen also showed that the pharmacological inhibitor of DYRK1A, harmine, rescues the cellular phenotypes in TgDYRK1A. Treatment with epigallocathecin 3-gallate (EGCG), a natural polyphenol component of green tea, which inhibits DYR$\mathrm{K} 1 \mathrm{~A}$, also rescues these cellular phenotypes and the behavioral and histological alterations in TgDYRK1A and improves memory in trisomic mice. Based on these and other data, clinical trials with EGCG are being pursued.

\section{HMGN1}

Michael Bustin (National Cancer Institute) discussed his work on high mobility group N1 protein (HMGN1) which binds to nucleosomes and affects the structure and function of chromatin. Genome wide, HMGN1 binds to numerous promoters where it co-localizes with DNaseI hypersensitive sites. Using a combination of transgenic overexpression and knockout models, he observed significant changes in gene expression upon altering HMGN1 levels. Overexpression of HMGN1 by 2 -fold led to dysregulation of more than 1,200 genes (454 decreased; 811 increased) suggesting that HMGN1 may profoundly affect global gene expression in DS. One of the genes that is negatively regulated by HMGN1 is methyl CpG binding protein 2 (MECP2), mutation of which leads to Rett syndrome, a neurodevelopmental disorder characterized by intellectual disability. Overexpression of HMGN1 led to reduced MeCP2 levels [Abuhatzira et al., 2011]. HMGN1 transgenics exhibited behavioral phenotypes consistent with other DS mouse models including hyperactivity, reduced anxiety and altered social interests. Thus, elevated HMGN1 levels in DS may lead to epigenetic changes that contribute to the observed complex neurological phenotypes.

\section{SYNJ1}

Fabian Corlier (Centre de Recherche de l'Institut du Cerveau et de la Moelle, France) reported the involvement of the phosphoinositol phosphatase SYNJ1 in the endosomal phenotype observed in DS. Previous work from their group demonstrated that SYNJ1 overexpression in DS lymphoblastoid cell lines (LCLs) contributed to endosome enlargement [Cossec et al., 2012]. Notably, these lines express low levels of APP, again suggesting that multiple HSA21 genes may contribute to endosomal abnormalities, as discussed earlier by R. Nixon. Using mutant forms of SYNJ1, Corlier showed that overexpression of either full-length SYNJ1 or a fragment containing a SAC1-like domain induced an increase in large endosomes and a decrease in small endosomes. This phenotype was similar to, but less severe than that observed in the Ts65Dn. Mutation of either the 5-phosphatase domain or the Pro-rich domains in SYNJ1 suppressed its ability to induce endosome enlargement when overexpressed in HEK293T cells. They further showed that enlargement of endosomes is more pronounced in LCLs derived from $\mathrm{DS}$ individuals with $\mathrm{AD}$ versus $\mathrm{DS}$ alone.

\section{Lessons from Other Models of Intellectual Disability}

\section{Fragile $X$ and Autism Spectrum Disorder}

De novo protein synthesis is a hallmark of long-term memory formation. The mTORC1 transduction pathway couples neurotransmission and neurotrophic signals to protein translation during long-lasting synaptic plasticity and the consolidation phase of long-term memory. Interestingly, mutations in upstream negative regulators and downstream effectors of $\mathrm{mTORC1}$, including fragile $\mathrm{X}$ mental retardation protein (FMRP) and eukaryotic translation initiation factor $4 \mathrm{E}$ (eIF4E), are associated with some forms of developmental intellectual disability (ID) and autism spectrum disorders (ASD). Eric Klann (New York University) presented results showing altered protein synthesis and abnormal translational control in the brains of fragile X (FX) model mice and EIF4E transgenic mice. Genetic reduction of the translational control molecule p70 S6 kinase (S6K1) in FX mice corrected altered protein synthesis and synaptic plasticity and aberrant behaviors. Similarly, pharmacological targeting of eIF4E in EIF4E transgenic mice reversed translational and synaptic abnormalities as well as ASD-associated behaviors. These results suggest that exaggerated protein synthesis triggers synaptic dysfunction and ASD-like behaviors in mice and identify translational control factors as novel therapeutic targets for the treatment of ASD.

\section{Immune System Function}

Jonathan Kipnis (University of Virginia) focused on the emerging role of immune cells in cognitive function and the relation between abnormal neuroimmune interactions and cognitive impairment. While it has been 
shown that $\mathrm{T}$ cells support learning and memory (L/M), the mechanisms are not clear. In experimental animals, performance of cognitive tasks leads to accumulation of IL-4-positive T cells and the activation of myeloid cells in meningeal spaces. Blockade of T-cell migration to meningeal spaces or elimination of $\mathrm{T}$ cells responding to CNS self-antigens result in impairment of $\mathrm{L} / \mathrm{M}$ and a skewed pro-inflammatory phenotype of meningeal myeloid cells. The role of IL-4 appears to be critical in this process since $I l 4^{-/-}$mice exhibit cognitive impairment and a similarly abnormal pro-inflammatory meningeal myeloid cell phenotype. These results point to a critical role for meningeal autoimmune $\mathrm{T}$ cells in the regulation of meningeal myeloid cells and cognitive function and underscore the potential of immune-based therapies for cognitive and mental diseases.

\section{Metabolomics}

Small molecules such as neurotransmitters, signal lipids, osmolytes, ions, and metabolites play fundamental signaling roles in the CNS. Thus, development of highthroughput metabolic profiling tools and metabolome research are particularly important in the CNS, where variations in brain metabolic homeostasis are associated with neurological disorders. Marc E. Dumas (Imperial College London) described the ongoing implementation of systems biology approaches to integrate metabolic data with protein interactions and pathway analysis to inform the structural and functional complexity of the brain in nor$\mathrm{mal}$ and pathological conditions. Using fragile $\mathrm{X}$ as a case study, networks were constructed from comparative metabolomics of control and $\mathrm{FMRP}^{-/-}$mouse brains, with the addition of known and predicted targets of FMRP, protein interaction partners and metabolomic and neuronal pathways of which they are components. The FMRP network was significantly shorter than expected from random associations. Interestingly, the network included HSA21 proteins APP and SOD1. It may be possible to extract information on perturbations in DS by querying this network for all HSA21 protein associations.

\section{Mitochondrial Function}

Jorge Busciglio (University of California, Irvine) described studies on LCLs generated from control, DS, and $\mathrm{DS} / \mathrm{AD}$ subjects, designed to assess the presence of metabolic deficits in peripheral cells associated with the development of AD in DS. The results indicate that both DS and DS/AD LCLs exhibit abnormal ATP levels and mitochondrial membrane potential compared to control LCLs. Interestingly, conditions that enforce the activa-

Conference on Cognition in Down Syndrome tion of mitochondria and oxidative phosphorylation (OXPHOS), such as galactose-based culture medium, lead to differential growth profiles between DS and DS/ AD LCLs, increased generation of free radicals and increased sensitivity to mitochondrial toxins specifically in DS LCLs. Thus, a differential glycolysis/OXPHOS balance between DS and DS/AD LCLs may represent a useful marker of dementia progression and/or treatment outcomes.

Pablo Helguera (Instituto Ferreyra, Argentina) focused on the relation between abnormal energy metabolism and oxidative stress with transcriptional and functional changes in DS cells. Sustained metabolic stimulation in DS cortical neurons and astrocytes restored mitochondrial functional parameters but also increased free radical levels and cell damage, suggesting that reduced mitochondrial activity in DS cells may be part of an adaptive response to avoid injury and preserve cellular functions. Network analysis of overexpressed genes in DS cells showed a close functional integration in energy metabolism- and oxidative stress-related pathways, underscoring the role of abnormal energy metabolism in DS. Thus, while mitochondrial downregulation appears to minimize free radical production and cellular injury, this adaptation may predispose individuals with DS to clinical conditions related to abnormal mitochondrial function such as $\mathrm{AD}$, diabetes and some forms of ASD.

\section{Mechanisms of ID in DS - Model Systems}

\section{Mouse}

Mouse has been an important model system for uncovering and analyzing phenotypic features of DS. Indeed it has a dominant place in DS research, in particular in investigation of neurological abnormalities and L/M impairments. Consistent with this, $60 \%$ of investigator-initiated abstracts at this conference discussed studies with one or more mouse models. These included the MMU16 segmental trisomies, Ts65Dn, Ts1Cje and Ts1Rhr, the HSA21 transchromosomal model Tc1, as well as several single gene overexpressing transgenic models (fig. 2).

Yann Herault (IGBMC, France) provided an overview of the 'aneuploid zoo', current segmental trisomy mouse models that are trisomic for differing, sometimes overlapping, sets of HSA21 orthologs [Herault et al., 2012]. The use of chromosome engineering with the Cre-lox system has made construction of these models possible. A new segmental trisomy is the Ts(5)Yah that is trisomic for the MMU16 segment spanning App to Runx1. These mice 
display normal $\mathrm{L} / \mathrm{M}$ in the Morris water maze and Novel Object Recognition tests.

Joseph Goodliffe (Boston University School of Medicine) described studies with the $\mathrm{Dp}(16) \mathrm{Yey}$, another Crelox engineered model, trisomic for the complete MMU16 region orthologous to HSA21. This model has important advantages with respect to the Ts65Dn: it is trisomic for the entire MMU16 segment orthologous to HSA21 and contains trisomy for no non-HSA21 orthologs. Prior reports have shown that, like the Ts65Dn, the Dp16 is impaired in several tests of L/M and displays abnormalities in long-term potentiation [Yu et al., 2010]. Goodliffe and colleagues further showed that Dp16 mice display developmental abnormalities similar to some seen in DS and the Ts65Dn, including microcephaly. Abnormalities are milder than in the Ts65Dn, possibly due to one of the following: (1) differences in chromatin arrangement due to the duplication of $22.9 \mathrm{Mb}$ to the end of chromosome 16 rather than a separate chromatid, (2) trisomy for the additional HSA21 orthologs in the Dp16 moderates phenotypic features, (3) the non-HSA21 orthologs trisomic in the Ts65Dn contribute to some of the phenotypic features or (4) strain differences.

Frances Wiseman (University College London Institute of Neurology) described further studies with the transchromosomal model, the Tc1, that carries an HSA21. In spite of some internal deletions [Gribble et al., 2013], the $\mathrm{Tcl}$ is currently the most complete DS model, trisomic for 121 of 161 HSA21 protein-coding genes. Wiseman and colleagues crossed the Tcl with a transgenic mouse carrying a mutation in the $A P P$ gene seen in some familial AD. Because the APP gene is not functionally trisomic in the $\mathrm{Tc} 1$, these experiments allow investigation of the potential contributions to $\mathrm{AD}$ in $\mathrm{DS}$ of additional HSA21 genes.

Md. Mahiuddin Ahmed (University of Colorado School of Medicine) described protein profiles in the $\mathrm{Dp}(10)$ Yey and Dp(17)Yey models. These mice are trisomic for HSA21 orthologous segments of MMU10 and 17, respectively [ $\mathrm{Yu}$ et al., 2010]. While neither has so far displayed deficits in L/M, Ahmed showed that these mice do show abnormalities in the levels of proteins known to be involved in $\mathrm{L} / \mathrm{M}$, including components of the MAP kinase and mTOR pathways, proteins encoded by immediate early genes, and several proteins related to Alzheimer's disease. Indeed, the protein profiles show many similarities to those seen in the Tc1 mouse model [Ahmed et al., 2013] which includes trisomy of both the MMU10 and MMU17 syntenic regions. These observations force questions regarding just what molecular abnormalities exist in the full trisomy HSA21 DS brain and what neurological defects the molecular abnormalities cause or reflect.

Damien Colas (Stanford University) analyzed features of sleep and EEG in the popular Ts65Dn. Treatment with pentylenetetrazole rescued $\mathrm{L} / \mathrm{M}$ deficits, but only if the drug was administered during the sleep phase. These findings add to the growing awareness that sleep abnormalities in DS may have important and broad contributions to cognitive function.

In other experiments with the Ts65Dn, Maria Usowicz (University of Bristol, UK) used whole-cell voltage clamp recordings to show that granule cells from the Ts65Dn have a reduced tonic $\mathrm{GABA}_{\mathrm{A}}$ receptor current density. Added to the overall reduced size of the cerebellum and reduced numbers of granule cells, altered electrophysiological properties may contribute to impaired motor and cognitive function in the Ts65Dn and in people with DS.

Damien Marechal (IGBMC, France) discussed the contribution of cystathione $\beta$-synthase (CBS) to the cognitive phenotypes of DS. The Ts(1)Yah mouse model (fig. 2), which is trisomic for HSA21 orthologs spanning Abcg1 to U2af1, including the Cbs gene, displays shortterm memory deficits [Pereira et al., 2009].

Nicole Creau (University of Paris Diderot, France) described cerebellar phenotypes in adult transgenic mice overexpressing the human Purkinje cell protein 4 (PCP4) gene. PCP4 expression was increased at all cell stages and neuronal compartments. While adult TgPCP4 mice showed normal cerebellar morphology, they were impaired on the rotarod and Morris water maze tests, indicating alterations in coordination and motor and procedural learning. In addition, electrophysiological recording in cerebellar Purkinje neurons revealed a 2 -fold increase in LTD in TgPCP4 mice compared to controls.

\section{Non-Mammalian in vivo Model Systems - \\ Caenorhabditis elegans}

While mouse is a required model system for analysis of $\mathrm{L} / \mathrm{M}$, the expense and time required for their use and manipulation are serious limitations, in particular for DS because of its complex genetic basis. Jon Pierce-Shimomura (University of Texas at Austin) described a project to use C. elegans as an alternative model organism. Specifically, RNAi can be used to knockdown individual genes and combinations of genes to uncover functional features of HSA21 orthologs. Of course, the evolutionary divergence of C. elegans from mammals and the presence of co-orthologs and paralogs means that functional conservation of highly similar proteins is not assured. Initial experiments 
overexpressing apl-1, the C. elegans ortholog of the human $A P P$ and APPL1, 2 genes, resulted in neurodegeneration and accumulation of APP in a subset of neurons.

Non-Mammalian in vivo Model Systems - Zebrafish

Sarah Edie (Johns Hopkins University) described use of zebrafish to determine functions of HSA21 genes. She chose cDNAs for 169 HSA21 genes, of which 149 have orthologs in mouse. These were transcribed in vitro and injected into 1-2-cell zebrafish embryos. Seven cDNAs, including those for superoxide dismutase (SOD1) and 2 novel open reading frames, C21orf84 and C21orf57, resulted in cyclopia and/or U-shaped somites, phenotypes related to decreased sonic-hedgehog (Shh) signaling. The phenotype showed variable penetrance, which was increased with co-injection of 4 cDNAs with C21orf 84 .

\section{Human in vitro Model Systems - Induced Pluripotent} Stem Cells

Mouse and other model systems have the limitations of allowing only partial trisomies and use of limited genetic background. Recent advances in induced pluripotent stem cell (iPSC) technology provide a cell system for investigating full HSA21 trisomy.

Anita Bhattacharyya (University of Wisconsin) generated iPSCs from 2 DS fibroblast lines. Because one line was a DS mosaic, 2 iPSC lines were obtained, 1 trisomic and 1 disomic for HSA21 [Weick et al., 2013]. This provides an important opportunity for direct comparison of the effects of trisomy HSA21 in an isogenic background. Trisomy neurons obtained from the iPSCs, but not the original iPSCs, showed oxidative stress. Microarray analysis indicated increased expression of HSA21 genes consistent with trisomy. Interestingly, trisomic neurons showed reduced synaptic activity equally affecting both excitatory and inhibitory transmission. This contrasts with observations in the Ts65Dn mice of reduced inhibitory neurotransmission only. Dean Nizetic (Queen Mary University of London) discussed a similar success in generating iPSCs from a mosaic DS fibroblast line.

As protocols for iPSC generation and differentiation become more robust, their widespread use will provide a system to examine the effects of genetic diversity on both neuronal phenotypes and drug responses, and their correlations with cognitive profiles of the donors of fibroblasts.

Human Model Systems - Reducing Trisomy to Disomy Targeting individual HSA21 genes with drugs to reduce their expression is clearly impractical given the number of genes likely to contribute to the DS cognitive phenotype and therefore the number of drugs required. Targeting downstream molecular abnormalities (e.g. decreased GABA transmission) will have some level of offtarget effect and may fail to address all important abnormalities. An alternative to controlling the consequences of trisomy is to develop 'chromosome therapy', i.e. to effectively remove or silence one HSA21 chromosome.

Li Li (University of Washington) described their approach which involved inserting a TKNEO cassette into the APP gene in a DS iPSC line. Selecting against TKNEO resulted in survival of only those cells that had lost neo expression [Li et al., 2012]. Although TKNEO deletion, point mutations and epigenetic silencing were observed, the most common mechanism of silencing was induced loss of the targeted HSA21. When trisomic and disomic cells were cultured together, the proportion of trisomic cells rapidly decreased due to their slower cell cycle and extended time in G1 phase.

Jeanne Lawrence (University of Massachusetts Medical School) approached the same problem through the molecular mechanism governing the normal process of $\mathrm{X}$ chromosome inactivation. Using a zinc finger nuclease targeting an HSA2 1 gene sequence, the $17-\mathrm{kb}$ XIST cDNA was inserted into an HSA2 1 in a DS iPSC line. When XIST expression was induced, the HSA21 chromosome was painted and molecular, cytological and genome analyses showed appropriate silencing of the corresponding HSA21 gene expression.

These approaches provide new options for comparing the effects of trisomy to disomy in in vitro isogenic cell lines. Importantly, while many challenges remain, they successfully demonstrate the first step required for developing chromosomal therapy for DS.

\section{Pharmacology in Model Systems}

\section{Ts65Dn}

More than a dozen drugs/small molecules have been shown to rescue $\mathrm{L} / \mathrm{M}$ and synaptic abnormalities in the Ts65Dn mouse model, but many details of drug responses remain to be assayed at the cell, molecular and physiological levels. In addition, little is known about the optimal age for treatment. Several presentations addressed some of these issues.

Carmen Martinez-Cue (University of Cantabria, Spain) described their detailed behavioral, electrophysiological and cellular analysis of Ts65Dn treated for 6 weeks with the selective $\mathrm{GABA}_{\mathrm{A}}$ a5 negative allosteric 
modulator, R04938581 [Martinez-Cue et al., 2013]. Not only did the drug rescue L/M deficits, it also normalized numbers of proliferating and mature granule cells in the dentate gyrus, reversed the deficit in long-term potentiation and suppressed hyperactivity. These positive effects were observed in the absence of negative effects on motor function, spontaneous locomotion, anxiety or seizure activity. These data support the potential therapeutic use of R04938581 in people with DS.

Katheleen Gardiner (University of Colorado School of Medicine) described response at the protein level of Ts65Dn mice exposed to context fear conditioning (CFC) with and without treatment with the NMDA receptor antagonist memantine. Memantine has previously been shown to rescue $\mathrm{L} / \mathrm{M}$ deficits in several tasks in the Ts65Dn, including CFC. Proteins, including components of MAP kinase, mTOR and PKC pathways, immediate early genes and NMDA receptor subunits, were measured in hippocampus and cortex of control and Ts65Dn mice. At baseline, i.e. without exposure to $\mathrm{L} / \mathrm{M}$ or memantine, compared to controls, Ts65Dn display elevated levels in several components of MAP kinase and mTOR pathways. In control mice, exposure to CFC produced changes in more than half of the proteins measured, but in the Ts65Dn, far fewer changes were seen. Treatment with memantine normalized some baseline protein levels in the Ts65Dn and facilitated further changes with CFC. Overall, these data show that successful L/M produces complex changes in protein profiles and that the Ts65Dn show complex patterns of abnormalities. Further experiments are needed to determine which abnormalities critically underlie L/M impairment and which are compensatory for trisomy perturbations.

Jean Delabar (University of Paris Diderot, France) expanded further on studies of the response of Ts65Dn to treatment with EGCG, an inhibitor of the HSA21-encoded protein kinase, DYRK1A. EGCG has been shown to rescue L/M deficits in the Ts65Dn and in the DYRK1ABAC transgenic. Delabar has now shown that EGCG also rescues abnormalities in levels of protein components of GABAergic and glutamatergic signaling pathways, consistent with regularization of ratios of excitatory and inhibitory transmission.

Early postnatal treatment with the serotonin reuptake inhibitor, fluoxetine, had been shown previously to rescue impairments in hippocampal neurogenesis and L/M in adult Ts65Dn mice [Bianchi et al., 2010]. Renata Bartesaghi (University of Bologna) extended these studies by treating pregnant Ts65Dn mice with fluoxetine from E10 until birth. Resulting Ts65Dn pups showed normal cell numbers at $\mathrm{P} 2$ in all brain regions examined, including the neocortex, hippocampus, striatum, and cerebellum. Furthermore, at 45 days of age with no postnatal treatment, Ts65Dn mice showed normal levels of neurogenesis, normal neuronal connectivity and no L/M impairment. While the ethical considerations of prenatal clinical trials for DS present challenges, evidence that prenatal pharmacotherapy can prevent neurodevelopmental and $\mathrm{L} / \mathrm{M}$ abnormalities caused by trisomy is an important advance.

\section{Combinatorial Pharmacotherapy}

Multiple drugs have shown efficacy in rescuing $\mathrm{L} / \mathrm{M}$ deficits in the Ts65Dn mice, however, clinical trials conducted so far for cognition in DS have shown at best modest success. While this may be due in part to small numbers of participants and the genetic limitations of the Ts65Dn mice used in preclinical evaluations, the possibility remains that effective therapeutics for human cognition may require multiple drugs to target multiple negative effects of trisomy HSA21. To this end, Chih-Ming Ho (University of California Los Angeles) presented a novel approach for rapid optimization of combinations of drugs. The method, Feedback System Control (FSC.X), combines direct experiment with computational prediction. An iterative, parallel search process alternates between using a computer algorithm to predict drug combinations, testing each combination experimentally and using the biological readout to predict new drug combinations for testing for improved efficacy. The method has been applied in cell culture to several biological paradigms, including viral infection and reactivation [Ding et al., 2012]. Notably, using combinations of 6 drugs, FSC.X converges to an optimum in only tens of searches rather than in hundreds of thousands needed to randomly sample the available parameter space. In addition, in the optimized combination, each component drug is effective at a concentration much lower than when used alone, thus decreasing the potential for negative side effects. The future of pharmacotherapy for cognition in DS may require consideration of drug combinations.

\section{The DS Cognitive and Neurological Phenotype}

George Capone (Kennedy Krieger Institute) addressed the topic of co-morbidities in children with DS, their potential impact on cognitive function and their implications for clinical trials. Common in children with DS are sleep disturbances and behavioral-mental health conditions, 
such as ASD, depression and attention deficit hyperactivity disorder. In considering clinical trials for cognition in DS, the presence of these potential saboteurs of cognitive function must be documented and controlled for. Failure to do so will confound data collection and interpretation, and possibly mask any real treatment response. No clinical trial in DS thus far has screened participants for all known secondary impairments. Importantly, sleep disturbances and neurobehavioral co-morbidities in DS have not been targeted specifically for treatment. Pharmacologic optimization of sleep and/or reduction of maladaptive behaviors might have a lasting and favorable effect on cognitive function in children with DS.

Len Abbeduto (MIND Institute) addressed language development in DS and its role in clinical trials. As pharmacological treatments for people with ID are increasingly condition-specific, evaluation of efficacy is constrained by a lack of adequate cognitive and behavioral outcome measures. Ideally, such measures should reflect syndrome-specific phenotypes and core deficits with functional value. Language is an ideal measurement for efficacy in DS because multiple impairments of expressive language are present. Structured expressive language sampling procedures would be preferable to standardized tests of language that are not designed to measure treatment efficacy.

Naznin Virji-Babul (University of British Columbia) spoke on perception-action coupling in DS, using data from magnetoencephalography in conjunction with behavior [Virji-Babul et al., 2011, 2012, 2013]. Cortical responses were measured during perceptual-motor processing associated with voluntary reaching versus observation of reaching, processing of emotional responses while listening to music and perception of facial expressions. Correspondence between the networks involved in action-execution and action-observation appears to be reduced in DS suggesting dysfunction in the mirror neuron system in relation to visual action representation. These deficits do not parallel deficits in audio-motor coupling, suggesting that independent networks mediate the visual and auditory representation of action. Understanding how individuals with DS process perceptual information may lead to novel methods for intervention.

Chitra Lal (Medical University of South Carolina) discussed the impact of obstructive sleep apnea syndrome (OSAS) on cognitive impairment. Cognitive domains most impacted by OSAS include vigilance, memory and executive function, which overlap with some features of DS. OSAS is common in DS and is characterized by significant hypoxemia, hypoventilation and sleep fragmen-

Conference on Cognition in Down

Syndrome tation, which tend to be more severe than in the non-DS population. The presence of cognitive impairment in DS may exacerbate the adverse impact of OSAS. Because OSAS is potentially reversible, prompt diagnosis and treatment of this co-morbidity may have a significant impact on the overall health and quality of life for persons with DS.

Ira Lott (University of California Irvine) discussed cognitive changes across the lifespan. Typically children with DS demonstrate greater morpho-syntax, verbal short-term and explicit long-term memory impairment compared with associative learning, visual-spatial shortterm and implicit long-term memory. Middle age appears to be a quiescent time for cognitive function. While dementia increases in individuals $>45$ years, it is not ubiquitous. Throughout the lifespan neuroinflammation, oxidative stress, amyloid accumulation, and medical comorbidities can impact cognitive function.

Nancy Raitano Lee (NIMH) described age effects on executive function (EF) in a multi-site study of youth with DS. Previously, in young children with DS, EF skills, such as regulation of emotion and behavior, were found to be relatively commensurate with mental age expectations. In contrast, working memory and planning/organizing were below mental age expectations. This is true also in older children and young adults with DS. While in typically developing children, there were statistically significant relationships between age and EF maturity in the domains of working memory and planning (suggesting improvements' with age in this cross-sectional sample), in the DS group (ages 5-25), significant age-EF relations were not found. Rather, the latter showed 'flatter' developmental trajectories, which is presumed to reflect slower rates of maturation. These findings provide further insight into the DS cognitive and behavioral phenotype in childhood.

Roger Reeves (Johns Hopkins University) discussed the need to establish a comprehensive genotype-phenotype study of DS called DS360. The goals are to define genetic contributions to the variability of individual phenotypic features and to establish co-morbidities of occurrence (penetrance) and severity (expressivity) among phenotypic features. Genetic analysis (GWAS, CNV, exome sequence and/or whole genome sequence) is needed with the data storage suitable for longitudinal studies. Linkage to a tissue repository would enable replication and further analyses of the transcriptome, proteome and epigenome, as well as provide a source of materials for iPSC generation. A virtual center structure is envisioned in which participating institutions follow consensus protocols for phenotyping. The current trans-NIH DS working group emphasizes 
the need for collaborative, inter-NIH Institute efforts to understand DS and the consequent implications for treatment of related conditions in all people.

\section{Clinical Trials for Cognition in DS - Panel Discussions}

There is now considerable enthusiasm for clinical trials for cognition in DS. This enthusiasm is based largely on successes in rescuing $\mathrm{L} / \mathrm{M}$ in the popular Ts65Dn mouse model of DS and involves a diverse array of drugs. Given the relative newness of clinical trials for cognition in DS, panel discussions were planned to highlight the important challenges and strategies for optimizing their success.

Michael Aman (Ohio State University) described lessons learned from clinical trials in other types of intellectual disability. Standard cognitive tests, e.g. Aberrant Behavior Checklist, Nisonger Scales or Clinical Global Impressions scale, may require adaptation for use in children with ID to ensure that appropriate mental age requirements are met and that there is sufficient sensitivity for detection of treatment effects. By looking to conditions other than DS, it may be possible to expand the existing array of test instruments to enhance evaluation.

Michael Harpold (DSRTF) raised several points that have concerned DS researchers in basic, clinical and translational fields for many years. These include the potential for biomarkers that may be promising and informative, and how to validate these for use in clinical trials as outcome measures in addition to cognitive testing. There is also the need to develop criteria to permit comparison of outcome measures between clinical trials that use different drugs, and criteria for prioritization of drug candidates for trials. Small clinical trials with young adults with DS have been reported using the NMDA antagonist, memantine, the HSA21 DYRK1A kinase inhibitor, EGCG, and folate. Trials are in progress for the $\mathrm{GABA}_{\mathrm{A}}$ antagonists R04938581 (O. Khwaja, below) and pentylenetetrazole, and for the combination of folate plus thyroxine. Completed trials have shown modest improvements, but also have used different outcome measures, making intertrial comparisons difficult. Discussion is also needed regarding what research is necessary to better ensure that drug treatment successes in preclinical models, whether mouse models or cell systems, will translate to efficacy in clinical trials.

Omar Khwaja (Hoffmann-LaRoche) described plans for a clinical trial that targets an imbalance between inhibitory and excitatory neurotransmission that is presumed to exist in the DS brain, based on observations in the Ts65Dn mouse. The imbalance is presumed to underlie or contribute to cognitive deficits in DS. $\mathrm{GABA}_{\mathrm{A}}$ receptor antagonists rescue cognitive and behavioral impairments by decreasing GABA-mediated inhibition. Roche has developed a highly selective $\mathrm{GABA}_{\mathrm{A}}$ a5 negative allosteric modulator (see presentation by C. Martinez-Cue) that lacks the proconvulsant property of the $\mathrm{GABA}_{\mathrm{A}}$ antagonist, pentylenetetrazole. Khwaja discussed issues such as the design of Phase 1-2 studies, outcome assessment strategy, safety considerations, biomarkers, operational issues, and regulatory strategy. Significant input from the academic and the DS community is required to best meet operational and scientific challenges in the development of treatment for cognitive and adaptive behavioral impairment.

The current unofficial standard for proposing utility in a clinical trial is the demonstration that a drug rescues $\mathrm{L} / \mathrm{M}$ in the Ts65Dn mouse model. This model is only a partial trisomy (fig. 2), lacking 3 copies of approximately $40 \%$ of HSA 21 protein-coding genes. Absent genes, including some discussed at this meeting, have functional properties of obvious relevance to DS phenotypic features. If absent genes influence phenotypic features and/ or drug responses, human clinical trials may continue to have at best questionable positive outcomes. An additional problem with reliance on mouse models is the limited genetic variability they provide, which again may be influencing drug responses and not reflecting responses that will occur most often in the genetically diverse human populations. The development of human cell systems, such as the neurally differentiated iPSCs discussed by A. Bhattacharyya and D. Nizetic, may provide an additional model system for drug efficacy evaluation that can address some aspects of complete trisomy and genetic heterogeneity.

G. Capone, L. Abbeduto, C. Lal and I. Lott expanded on their presentations regarding aspects of the DS cognitive and neurological phenotypic features. They discussed how these should be documented in participants in clinical trials (e.g. neurobehavioral co-morbidities) or ameliorated prior to a participant's enrollment in a clinical trial (e.g. sleep disturbances), how they can be incorporated as new outcome measures (e.g. language), and how outcome measures should be varied depending on age of participants.

\section{Summary}

As information on the functions of HSA21 genes continues to accumulate, so do hypotheses regarding how each may contribute to the cognitive phenotype in 
DS. Human model systems now available in neurally differentiated iPSCs provide new opportunities to study the molecular and cellular abnormalities present in full trisomy HSA21, their responses to drug treatments, and how all of these vary among individuals with DS. The potential for effective pharmacotherapy for cognition in DS is real. Still to be determined, however, are issues of optimal preclinical evaluation of drug efficacy and optimal design of clinical trials, including identification of practical and informative outcome measures. Also required is clear communication between basic, clinical and translational researchers and people with DS, their families and their physicians regarding the challenges that remain before pharmacotherapies become realities.

\section{Acknowledgement}

The conference was made possible by support from the Fondation Jérôme Lejeune, the Global Down Syndrome Foundation, F. Hoffman-LaRoche, the National Institutes of Health (U13HD075580), the Down Syndrome Research and Treatment Foundation, the Association Française pour la Recherche sur la Trisomie 21, Research Down Syndrome and Fresh Eyes Editing.

\section{References}

Abuhatzira L, Shamir A, Schones DE, Schäffer AA, Bustin M: The chromatin-binding protein HMGN1 regulates the expression of methyl CpG-binding protein 2 (MECP2) and affects the behavior of mice. J Biol Chem 286: 42051-42062 (2011).

-Ahmed MM, Dhanasekaran AR, Tong S, Wiseman FK, Fisher EM, et al: Protein profiles in Tc1 mice implicate novel pathway perturbations in the Down syndrome brain. Hum Mol Genet 22:1709-1724 (2013).

-Bianchi P, Ciani E, Guidi S, Trazzi S, Felice D, et al: Early pharmacotherapy restores neurogenesis and cognitive performance in the Ts65Dn mouse model for Down syndrome. J Neurosci 30:8769-8779 (2010).

-Ceru S, Konjar S, Maher K, Repnik U, Krizaj I, et al: Stefin B interacts with histones and cathepsin L in the nucleus. J Biol Chem 285:1007810086 (2010).

- Cossec JC, Lavaur J, Berman DE, Rivals I, Hoischen $\mathrm{A}$, et al: Trisomy for synaptojanin 1 in Down syndrome is functionally linked to the enlargement of early endosomes. Hum Mol Genet 21:3156-3172 (2012).

Ding X, Sanchez DJ, Shahangian A, Al-Shyoukh I, Cheng G, Ho CM: Cascade search for HSV1 combinatorial drugs with high antiviral efficacy and low toxicity. Int J Nanomedicine 7: 2281-2292 (2012).

-Duchon A, Raveau M, Chevalier C, Nalesso V, Sharp AJ, Herault Y: Identification of the translocation breakpoints in the Ts65Dn and Ts1Cje mouse lines: relevance for modeling Down syndrome. Mamm Genome 22:674684 (2011).

- Gribble SM, Wiseman FK, Clayton S, Prigmore E, Langley E, et al: Massively parallel sequencing reveals the complex structure of an irradiated human chromosome on a mouse background in the Tc1 model of Down syndrome. PLoS One 8:e60482 (2013).
Herault Y, Duchon A, Velot E, Maréchal D, Brault $\mathrm{V}$ : The in vivo Down syndrome genomic library in mouse. Prog Brain Res 197:169-197 (2012).

Hunter MP, Nelson M, Kurzer M, Wang X, Kryscio RJ, et al: Intersectin 1 contributes to phenotypes in vivo: implications for Down's syndrome. Neuroreport 22:767-772 (2011).

Li LB, Chang KH, Wang PR, Hirata RK, Papayannopoulou T, Russell DW: Trisomy correction in Down syndrome induced pluripotent stem cells. Cell Stem Cell 11:615-619 (2012).

Martínez-Cué C, Martínez P, Rueda N, Vidal R, García S, et al: Reducing $\mathrm{GABA}_{\mathrm{A}}$ a5 receptormediated inhibition rescues functional and neuromorphological deficits in a mouse model of Down syndrome. J Neurosci 33:39533966 (2013).

Pereira PL, Magnol L, Sahún I, Brault V, Duchon A, et al: A new mouse model for the trisomy of the Abcg1-U2af1 region reveals the complexity of the combinatorial genetic code of Down syndrome. Hum Mol Genet 18:47564769 (2009).

Russo A, O'Bryan JP: Intersectin 1 is required for neuroblastoma tumorigenesis. Oncogene 31: 4828-4834 (2012).

Schupf N, Zigman WB, Tang MX, Pang D, Mayeux $R$, et al: Change in plasma $A \beta$ peptides and onset of dementia in adults with Down syndrome. Neurology 75:1639-1644 (2010).

-Shapiro LA, Bialowas-McGoey LA, WhitakerAzmitia PM: Effects of S100B on serotonergic plasticity and neuroinflammation in the hippocampus in Down syndrome and Alzheimer's disease: studies in an S100B overexpressing mouse model. Cardiovasc Psychiatry Neurol 2010:pii153657 (2010).

Sosa LJ, Bergman J, Estrada-Bernal A, Glorioso TJ, Kittelson JM, Pfenninger KH: Amyloid precursor protein is an autonomous growth cone adhesion molecule engaged in contact guidance. PLoS One 8:e64521 (2013).
Sturgeon X, Gardiner KJ: Transcript catalogs of human chromosome 21 and orthologous chimpanzee and mouse regions. Mamm Genome 22:261-271 (2011).

-Virji-Babul N, Moiseev A, Moiseeva N, Sun W, Ribary U, Lott I: Altered brain dynamics during voluntary movement in individuals with Down syndrome. Neuroreport 22:358-364 (2011).

Virji-Babul N, Watt K, Nathoo F, Johnson P: Recognition of facial expressions of emotion in adults with Down syndrome. Phys Occup Ther Pediatr 32:333-343 (2012).

Virji-Babul N, Moiseev A, Sun W, Feng T, Moiseeva N, et al: Neural correlates of music recognition in Down syndrome. Brain Cogn 81: 256-262 (2013).

-Weick JP, Held DL, Bonadurer GF 3rd, Doers ME, Liu Y, et al: Deficits in human trisomy 21 iPSCs and neurons. Proc Natl Acad Sci USA 110:9962-9967 (2013).

Wilming LG, Gilbert JG, Howe K, Trevanion S, Hubbard T, Harrow JL: The vertebrate genome annotation (Vega) database. Nucleic Acids Res 36 (Database issue):D753-760 (2008).

Xie YF, Belrose JC, Lei G, Tymianski M, Mori Y, et al: Dependence of NMDA/GSK-3 $\beta$ mediated metaplasticity on TRPM2 channels at hippocampal CA3-CA1 synapses. Mol Brain 4:44 (2011).

Yang DS, Stavrides P, Mohan PS, Kaushik S, Kumar A, et al: Therapeutic effects of remediating autophagy failure in a mouse model of Alzheimer disease by enhancing lysosomal proteolysis. Autophagy 7:788-789 (2011).

-Yu T, Liu C, Belichenko P, Clapcote SJ, Li S, et al: Effects of individual segmental trisomies of human chromosome 21 syntenic regions on hippocampal long-term potentiation and cognitive behaviors in mice. Brain Res 1366: 162-171 (2010). 


\section{Erratum}

Cytogenetic and

Genome Research

In the article by Busciglio J, et al., entitled 'Down syndrome: genes, model systems, and progress towards pharmacotherapies and clinical trials for cognitive deficits' [Cytogenet Genome Res 2013;141:260-271, DOI: 10.1159/000354306], the name of John O’Bryan was unfortunately misspelled. 